\title{
(2) OPEN ACCESS \\ Relationship between emergency department and inpatient occupancy and the likelihood of an emergency admission: a retrospective hospital database study
}

\author{
Steven Wyatt (10, ${ }^{1}$ Ruchi Joshi, ${ }^{2}$ Janet M Mortimore, ${ }^{3,4}$ Mohammed A Mohammed ${ }^{1,5}$
}

Handling editor Loren De Freitas

Additional supplementa material is published online only. To view, please visit the journal online (http://dx.doi. org/10.1136/emermed-2021211229)

'Strategy Unit, NHS Midlands and Lancashire Commissioning Support Unit, West Bromwich, UK

2Emergency Department, Walsall Healthcare NHS Trust, Walsall, UK

${ }^{3}$ Faculty of Education,

Health and Wellbeing,

University of Wolverhampton,

Wolverhampton, UK

${ }^{4}$ Performance and Information

Team, Walsall Healthcare NHS

Trust, Walsall, UK

${ }^{5}$ Faculty of Health Studies,

University of Bradford, Bradford, UK

\section{Correspondence to}

Steven Wyatt, Strategy Unit, NHS Midlands and Lancashire Commissioning Support Unit, West Bromwich B70 9LD, UK; swyatt@nhs.net

Received 26 January 2021 Accepted 9 July 2021

\section{Check for updates}

(C) Author(s) (or their employer(s)) 2021. Re-use permitted under CC BY-NC. No commercial re-use. See rights and permissions. Published by BMJ.

To cite: Wyatt $S$, Joshi $R$, Mortimore JM, et al. Emerg Med J Epub ahead of print: [please include Day Month Year]. doi:10.1136/ emermed-2021-211229

\begin{abstract}
Background We investigate whether admission from a consultant-led ED is associated with ED occupancy or crowding and inpatient (bed) occupancy.
\end{abstract}

Methods We used general additive logistic regression to explore the relationship between the probability of an ED patient being admitted, ED crowding and inpatient occupancy levels. We adjust for patient, temporal and attendance characteristics using data from 13 English NHS Hospital Trusts in 2019. We define quintiles of occupancy in ED and for four types of inpatients: emergency, overnight elective, day case and maternity. Results Compared with periods of average occupancy in $E D$, a patient attending during a period of very high (upper quintile) occupancy was 3.3\% less likely (relative risk (RR) $0.967,95 \% \mathrm{Cl} 0.958$ to 0.977 ) to be admitted, whereas a patient arriving at a time of low ED occupancy was $3.9 \%$ more likely (RR $1.03995 \%$ Cl 1.028 to 1.050 ) to be admitted. When the number of overnight elective, day-case and maternity inpatients reaches the upper quintile then the probability of admission from ED rises by $1.1 \%$ (RR $1.01195 \%$ Cl 1.001 to 1.021), 3.8\% (RR $1.03895 \% \mathrm{Cl}$ 1.025 to 1.051 ) and $1.0 \%$ (RR $1.01095 \% \mathrm{Cl} 1.001$ to $1.020)$, respectively. Compared with periods of average emergency inpatient occupancy, a patient attending during a period of very high emergency inpatient occupancy was $1.0 \%$ less likely (RR $0.99095 \% \mathrm{Cl} 0.980$ to 0.999 ) to be admitted and a patient arriving at a time of very low emergency inpatient occupancy was $0.8 \%$ less likely (RR $0.99295 \% \mathrm{Cl} 0.958$ to 0.977 ) to be admitted.

Conclusions Admission thresholds are modestly associated with ED and inpatient occupancy when these reach extreme levels. Admission thresholds are higher when the number of emergency inpatients is particularly high. This may indicate that riskier discharge decisions are taken when beds are full. Admission thresholds are also high when pressures within the hospital are particularly low, suggesting the potential to safely reduce avoidable admissions.

\section{INTRODUCTION}

In the NHS, the main route to a hospital bed in an emergency is via an ED. ${ }^{1}$ A recent paper highlighted that case-mix-adjusted admission thresholds via ED increased considerably in recent years such that patients with similar risk profiles are now less likely to be admitted than a few years ago. ${ }^{2}$ It
Key messages

What is already known on this subject

- The likelihood of a patient being admitted via the ED is known to vary with patient case-mix factors and other factors such as arrival mode (eg, ambulance vs walk-in).

- Less is known about the extent to which the decision to admit is influenced by how busy the ED or the hospital is.

- Understanding this relationship might suggest opportunities to improve patient safety and enhance hospital efficiency.

What this study adds

- This study uses patient-level data and mixedeffects general additive logistic regression to assess the extent to which ED crowding and inpatient (bed) occupancy levels influence admission thresholds via ED in 13 English NHS Hospital Trusts in 2019.

- The probability of emergency admission from ED corresponds modestly to crowding and occupancy, reducing as ED gets busier and increasing when the number of elective and maternity inpatients is high or when the number of emergency inpatients is particularly high or low.

- Further work is required to uncover the underlying mechanisms, understand the implications for patient safety, hospital efficiency and generalisability of these findings.

could be argued that increases in admission thresholds via ED indicate that policy and service interventions to reduce avoidable admissions may be taking effect. The NHS in England, like many other health systems, has for some time sought to reduce avoidable admissions. But others have suggested increases in admission thresholds may be driven by a lack of hospital beds. This might undermine patient safety if patients are discharged when their clinical condition warrants admission. ${ }^{3}$ The bed availability argument is supported by data showing increases in bed occupancy in the NHS in England over recent years. ${ }^{4}$ The issue of ED crowding, which is often attributed to lack of inpatient beds, continues to receive considerable attention. Studies 
have demonstrated that crowding in the ED leads to delays in care and poor outcomes. ${ }^{5}$

The probability that a patient in an ED is admitted to a ward or assessment unit is known to vary according to several patient and attendance characteristics (eg, diagnosis, arrival mode, age). However, less is known about the relationship between the probability of admission via ED and the busyness of the hospital at that time. If, having adjusted for case-mix, admission thresholds vary according to ED crowding levels or inpatient occupancy, then this might indicate the circumstances when more risky discharge decisions are taken or when EDs are more able to safely avoid unnecessary admissions.

In this paper, we explore the extent to which ED crowding and inpatient (bed) occupancy levels are associated with admission thresholds via ED in 13 English NHS Hospital Trusts in 2019.

\section{METHODS}

\section{Study design, setting and population}

We conducted a retrospective cross-sectional analysis of 1314 942 attendances at consultant-led EDs in 13 NHS Hospital Trusts (providers) located in England. The providers are located throughout England and have a combined catchment population of 4.8 million people. ${ }^{6}$ Some of the 13 providers deliver care from more than one site.

The attendances took place between 1 January 2019 and 31 December 2019. Follow-up attendances were excluded along with attendances for patients who were dead on arrival, who died in the department or who left the department having refused treatment or before being treated $(n=80$ 850, 5.6\%). Records without a valid age, gender, resident lower super output area were also excluded from the analysis $(n=36908,2.6 \%)$.

\section{Variables and data sources}

Our analysis was based on three datasets held in the National Commissioning Data Repository. These datasets are commonly pseudonymised such that variables which serve to identify the patient (eg, name, addresses, NHS number, etc) are replaced with a coded pseudonym, with a consistent pseudonym used for a given patient in all three datasets. Dataset 1 contained information about attendances at EDs. Dataset 2 contained information about admitted patient care and dataset 3 contained supplementary information about the dates and times that patients were admitted to, discharged from and moved between inpatient units and wards within a hospital. The structure and content of these tables are described in the National Commissioning Data Repository Reference Library. $^{7}$

Because the datasets were commonly pseudonymised, we were able to identify whether the patient had recently attended the ED or indeed any other consultant-led ED and the outcome of this attendance.

Our outcome variable was admission from ED (yes/no). The outcome variable was defined by linking a patient's ED attendance record in dataset 1 , with inpatient records in dataset 2 to search for an emergency admission for the patient occurring on the same day as the discharge from ED.

Potential explanatory variables were identified with reference to previous studies and included patient characteristics (age, gender, ethnicity, deprivation, primary diagnosis, acuity, prior ED attendances and admissions), arrival mode, by ambulance or other means, and the month of year, day of week and time of day of arrival which were available in dataset 1 .
Patient ethnicity was assembled into six groups: white, Asian/Asian British, black/black British, mixed parentage, other ethnic groups and not known/not stated. Socioeconomic status was measured using the 2015 Index of Multiple Deprivation (IMD) rank assigned to the lower super output area in which the patient lived. ${ }^{8}$ IMD ranks were grouped into quintiles. A patient's prior ED activity was assigned to three levels: none, attended ED at least once but not admitted via ED, and attended and admitted at least once. Prior ED activity levels were assigned for two time periods: the 28 days before attendance and between 29 and 365 days before attendance (dataset 1 contained information about attendances in the 12 months prior to our study period). Thirty-nine two-digit primary ED diagnosis classification codes were used to define the patient's diagnosis in ED. Arrival month, arrival weekday and arrival hour were derived from the arrival date and time fields. Direct observations of patient acuity were not consistently available over the study period.

Dataset 1 was also used to calculate the number of patients in each trust's ED at 15-minute intervals over the course of the study period. This was achieved by counting patients who had arrived in the department before the end of each 15-minute period and left after the start of the period. Similarly, datasets 2 and 3 were used to calculate the number of patients in inpatient beds at 15-minute intervals, by admission method (overnight elective, day case, emergency, maternity). These measures of ED and inpatient occupancy, our variables of interest, were assigned to each attendance based on the start time of treatment in ED or the arrival time in ED if treatment start time was not available.

\section{Statistical methods}

Mixed-effects general additive logistic regression was used to explore the shape of the relationship between ED and inpatient occupancy and the probability of admission having adjusted for a range of patients and attendance characteristics. ${ }^{9}$

All candidate predictor variables were identified from prior literature ${ }^{2}$ and were included in a preliminary model on the basis of adequately strong univariate association with the outcome variable or because inclusion improved the fit of the multivariable model. The relationship between age and admission appeared non-linear and so was centred and entered into the model as a smoothed (thin plate spline) term. Several variables (ethnicity, deprivation, prior activity in the previous 28 and 29-365 days, diagnosis, arrival month, arrival weekday, arrival hour, provider trust) were recoded as design (dummy) variables. We tested the impact of including a small number of plausible variable interactions.

Our preliminary model included the following variables: age, sex, deprivation quintile, ethnicity, presenting diagnosis, arrival mode, arrival weekday, arrival hour, arrival month, prior attendances and admissions in the last 28 and 29-365 days, numbers of patients in ED, and the number of emergency, maternity, overnight elective and elective day-case inpatients. Provider trust was included in the model as a random effect. The preliminary model also included two interaction terms: age-gender and arrival mode-diagnosis.

Once we had constructed a well-fitting preliminary model using the patient and attendance variables, we sought to introduce our novel predictors of interest, measures of ED and inpatient occupancy. These variables were first centred and scaled within each provider trust and tested as linear covariates and as general additive (or smoothed) terms. These took the form of thin plate splines. 
Decisions about which variables to include in the final model were taken with reference to the significance of the model coefficients, the Wald test, the log-likelihood value, the Bayesian information criterion and the unbiased risk estimator. The final model fit was measured using the C-statistic (area under the receiver operating characteristic curve), calibration plots and the Hosmer-Lemeshow goodness-of-fit test.

While general additive models are flexible and can illustrate the (non-linear) shape of the relationship between an independent and dependent variable, the strength of the relationship between smoothed independent and dependent variables can be difficult to interpret. To overcome this issue, we built a second model, transforming the ED and inpatient occupancy variables into quintiles to represent five levels of busyness in $\mathrm{ED}$ and on inpatient wards by type of admission (elective, day case, emergency and maternity). We devised five occupancy quintiles (very high occupancy, above average occupancy, average occupancy, below average occupancy, very low occupancy) for ED crowding and inpatient occupancy. These design variables replaced the smoothed occupancy terms of the primary model. The model coefficients for these design variables were used to illustrate the significance and strength of the impact of high and low occupancy levels on the probability of admission.

The model covariates for time of day, day of week and month of year were used to adjust for the usual temporal patterns of busyness in as much as they influence whether a patient is admitted or not. The occupancy terms therefore estimate the effects of busyness over and above these usual patterns.

Effects sizes were converted to average relative risks using the approach described by Grant. ${ }^{10}$

To illustrate the materiality of our findings, we use this model to predict the number of patients in ED that would be admitted under occupancy conditions (quintiles) that are most conducive to admission and discharge.

Data processing was conducted in Microsoft SQL Server Management Studio V.17.5 and analysis in R V.3.5.1 ${ }^{11}$ and several R packages: tidyverse, broom, lmtest, PredictABEL, ResourceSelection and mgcv.

\section{RESULTS}

\section{Description of ED attendances}

Over the 12-month study period, there were 1314942 eligible ED attendances at the study sites, $33.2 \%$ of which were admitted (table 1). The distribution of attendances by age and sex was broadly similar to those for the country as a whole, but attendances of people from black and minority ethnic groups, more deprived areas and from the north east and north west were marginally under-represented (see online supplemental file 1).

A higher proportion of female attendances were admitted $(34.1 \%$ vs $32.4 \%)$. The proportion of patients who were admitted increased with age. A total of $30.5 \%$ of patients arrived by ambulance and more than half (58.6\%) of these patients were admitted. Patients attending with illness presentations (eg, cardiac, respiratory, gastrointestinal conditions) were considerably more likely to be admitted than patients with injury presentations.

The average (mean) number of patients in ED over the study period was 647 (SD 198). Mean ED occupancy varied across the 13 providers from 19 to 79 patients. There were on average 11065 emergency inpatients over the study period (provider range 509-1285), 894 overnight elective inpatients (provide range 23-204), 282 elective day-case inpatients (provider range

\begin{tabular}{|c|c|c|c|c|}
\hline All attendances & - & 1314942 & $(100.0 \%)$ & $33.2 \%$ \\
\hline \multirow[t]{2}{*}{ Sex } & Female & 676281 & $(51.4)$ & 34.1 \\
\hline & Male & 638661 & $(48.6)$ & 32.4 \\
\hline \multirow[t]{5}{*}{ Age group } & $0-14$ years & 284479 & (21.6) & 18.7 \\
\hline & $15-34$ years & 303026 & $(23.0)$ & 21.7 \\
\hline & $35-54$ years & 255491 & (19.4) & 28.3 \\
\hline & $55-74$ years & 241292 & (18.4) & 42.9 \\
\hline & $75+$ years & 230654 & $(17.5)$ & 61.8 \\
\hline \multirow[t]{2}{*}{ Arrival mode } & Ambulance & 400411 & $(30.5)$ & 58.6 \\
\hline & Walk-in & 914531 & $(69.5)$ & 22.2 \\
\hline \multirow[t]{8}{*}{ Diagnosis } & Cardiac conditions & 69152 & (5.3) & 60.0 \\
\hline & Contusion/abrasion & 80584 & (6.1) & 6.6 \\
\hline & $\begin{array}{l}\text { Dislocation/ } \\
\text { fracture/joint injury/ } \\
\text { amputation }\end{array}$ & 91706 & $(7.0)$ & 21.4 \\
\hline & $\begin{array}{l}\text { Gastrointestinal } \\
\text { conditions }\end{array}$ & 100024 & (7.6) & 45.2 \\
\hline & $\begin{array}{l}\text { Respiratory } \\
\text { conditions }\end{array}$ & 117785 & $(9.0)$ & 42.9 \\
\hline & $\begin{array}{l}\text { Soft tissue } \\
\text { inflammation }\end{array}$ & 26458 & $(2.0)$ & 22.1 \\
\hline & $\begin{array}{l}\text { Urological } \\
\text { conditions }\end{array}$ & 60055 & $(4.6)$ & 48.0 \\
\hline & Other/not known & 769178 & $(58.5)$ & 31.2 \\
\hline \multirow[t]{13}{*}{ Provider } & Provider 1 & 146530 & $(11.1)$ & 27. \\
\hline & Provider 2 & 104195 & (7.9) & 33.9 \\
\hline & Provider 3 & 86816 & (6.6) & 31.2 \\
\hline & Provider 4 & 109529 & (8.3) & 46.5 \\
\hline & Provider 5 & 102475 & (7.8) & 35.7 \\
\hline & Provider 6 & 133043 & $(10.1)$ & 30.0 \\
\hline & Provider 7 & 47689 & (3.6) & 33.3 \\
\hline & Provider 8 & 76986 & $(5.9)$ & 32.2 \\
\hline & Provider 9 & 105800 & (8.0) & 29.3 \\
\hline & Provider 10 & 43348 & (3.3) & 43.2 \\
\hline & Provider 11 & 136909 & $(10.4)$ & 35.0 \\
\hline & Provider 12 & 109969 & (8.4) & 33.1 \\
\hline & Provider 13 & 111653 & (8.5) & 29.6 \\
\hline
\end{tabular}

10-35) and 454 maternity inpatients (provider range 11-58) (see table B of online supplemental file 2).

Counts of patients in ED and the number of inpatients varied systematically by time of day, day of week and month of year (see figure 3 of online supplemental file 2). There was nonetheless considerable residual variation over and above these temporal patterns.

\section{Model fit}

Our final model estimated the probability of a patient being admitted using the following variables: patient's age, gender, ethnicity, deprivation, primary diagnosis, prior ED attendances and admissions, arrival mode, month of year, day of week and time of day of arrival, the numbers of patients in ED, and the number of emergency, maternity and elective and day-case inpatients at the start of treatment.

The C-statistic for the model was 0.831. A full set of the model covariates and coefficients are included as online supplemental file 3. A calibration plot and the receiver operating characteristic curve are shown in online supplemental file 4. 


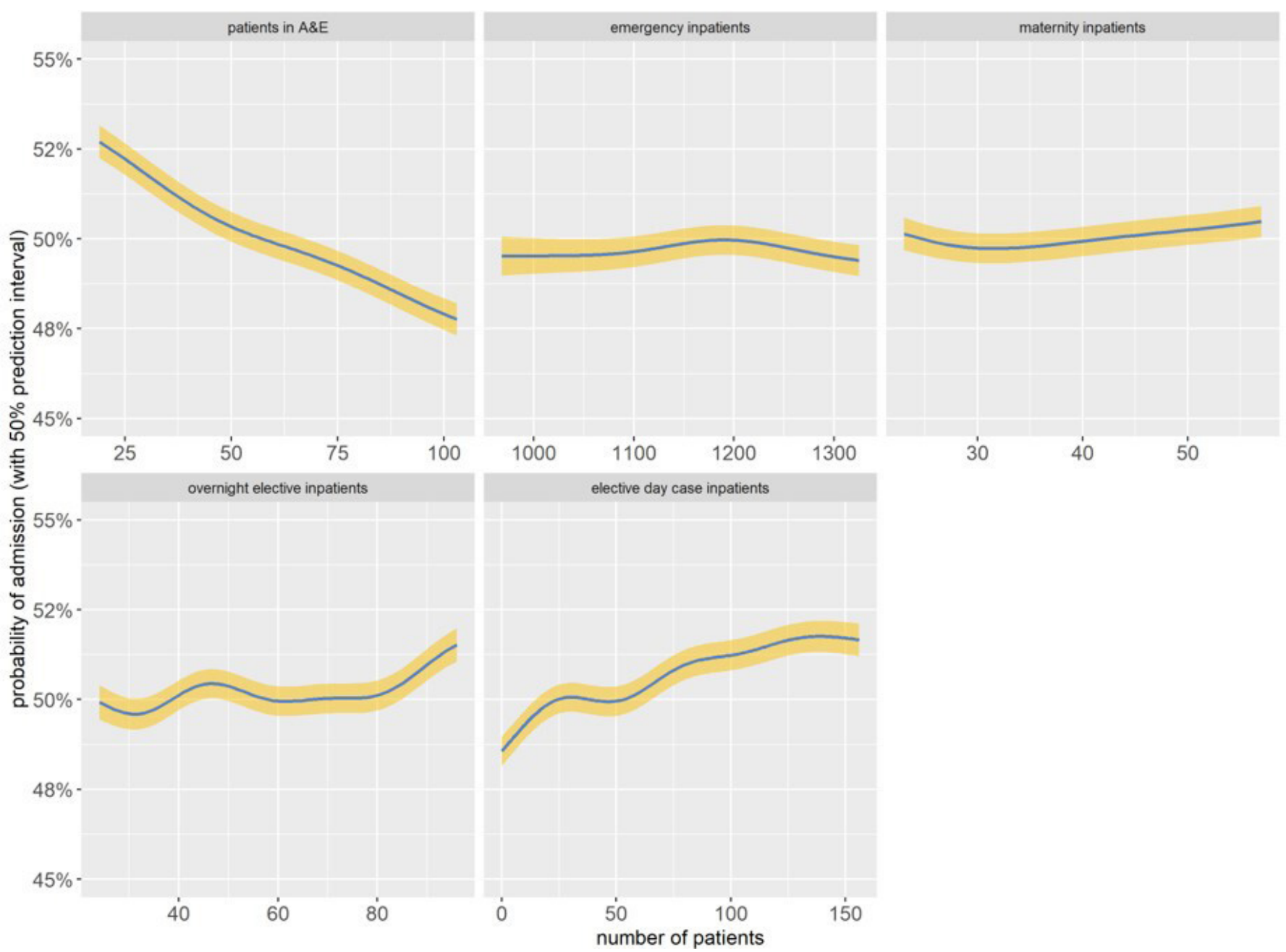

Figure 1 The relationship between ED and inpatient occupancy and the probability of admission; shaded area indicates $50 \%$ prediction interval.

\section{The relationship between ED crowding and inpatient occupancy on admission thresholds}

We illustrate the relationship between ED and emergency inpatient occupancy and the probability of admission, with respect to an exemplar patient; a 65 -year-old white man who lives in a deprived area (IMD 2015 quintile 1), who presents via walk-in at provider 12 with a cardiac condition at 16:00 on a Monday in October, with no previous attendance in the last year. This case is selected because under average ED and emergency inpatient occupancy conditions given the time of day, day of week and month of year, the modelled probability of admission for such a patient is $50 \%$.

Figure 1 illustrates how this probability of admission varies under alternative ED and inpatient occupancy conditions. The adjusted probability of admission via ED falls in a broadly linear fashion as the number of patients in ED rises. The adjusted probability of admission peaks around 1200 emergency inpatients but falls marginally in a non-linear fashion on either side of this. The adjusted probability of admission rises marginally with the number of maternity inpatients. The adjusted probability of admission rises as the number of overnight elective patients exceeds 80 patients and in a broadly linear fashion with the number of day-case patients.

To illustrate the relationship between ED and inpatient occupancy and the probability of admission in more general terms, we developed a second model in which the five smoothed patient occupancy variables (ED patients, emergency inpatients, maternity inpatients, overnight elective inpatients and elective daycase inpatients) were each divided into five quintiles of busyness (very high occupancy, above average occupancy, average occupancy, below average occupancy, very low occupancy). Table 2 and figure 2 contains the model covariates for each of the occupancy levels, having adjusted for all other terms in the model. The adjusted ORs range from 0.95 to 1.06 indicating modest effects.

\section{DISCUSSION Key findings}

Our study explored the relationship between ED and inpatient occupancy and admission thresholds. We found that admission thresholds are not fixed, but rather appear to correspond modestly to levels of ED and inpatient occupancy levels. These effects are limited to times when occupancy and crowding reach extreme levels. Under normal circumstances, clinical judgements do not appear to be affected by the context within which the decision is taken. But there is evidence that admission decisions are modified somewhat when EDs and hospitals become unusually busy or quiet.

After adjusting for patient and attendance characteristics including time of day and day of week, the 'same' patient is more likely to be admitted when ED occupancy is low and when 
Table 2 Adjusted OR and average relative risks of admission by ED and inpatient occupancy quintiles

\begin{tabular}{|c|c|c|c|c|c|}
\hline Term & Quintile & Adjusted OR & $95 \% \mathrm{Cl}$ & $\begin{array}{l}\text { Adjusted average relative } \\
\text { risks }\end{array}$ & $95 \% \mathrm{Cl}$ \\
\hline \multirow[t]{5}{*}{ ED occupancy quintiles } & 1-very low occupancy & 1.060 & 1.042 to 1.077 & 1.039 & 1.028 to 1.050 \\
\hline & 2-below average occupancy & 1.013 & 0.999 to 1.027 & 1.009 & 0.999 to 1.018 \\
\hline & 3-average occupancy (ref) & 1.000 & & 1.000 & \\
\hline & 4-above average occupancy & 0.983 & 0.969 to 0.997 & 0.988 & 0.979 to 0.998 \\
\hline & 5-very high occupancy & 0.952 & 0.938 to 0.966 & 0.967 & 0.958 to 0.977 \\
\hline \multirow[t]{5}{*}{ Emergency inpatient occupancy quintiles } & 1 -very low occupancy & 0.988 & 0.974 to 1.002 & 0.992 & 0.982 to 1.001 \\
\hline & 2-below average occupancy & 1.008 & 0.994 to 1.022 & 1.005 & 0.996 to 1.014 \\
\hline & 3-average occupancy (ref) & 1.000 & & 1.000 & \\
\hline & 4-above average occupancy & 0.996 & 0.983 to 1.010 & 0.998 & 0.988 to 1.007 \\
\hline & 5-very high occupancy & 0.985 & 0.970 to 0.999 & 0.990 & 0.980 to 0.999 \\
\hline \multirow[t]{5}{*}{ Maternity inpatient occupancy quintiles } & 1 -very low occupancy & 0.991 & 0.977 to 1.005 & 0.994 & 0.985 to 1.003 \\
\hline & 2-below average occupancy & 1.003 & 0.989 to 1.017 & 1.002 & 0.993 to 1.011 \\
\hline & 3-average occupancy (ref) & 1.000 & & 1.000 & \\
\hline & 4-above average occupancy & 1.007 & 0.994 to 1.022 & 1.005 & 0.996 to 1.014 \\
\hline & 5-very high occupancy & 1.015 & 1.001 to 1.030 & 1.010 & 1.001 to 1.020 \\
\hline \multirow{5}{*}{$\begin{array}{l}\text { Overnight elective inpatient occupancy } \\
\text { quintiles }\end{array}$} & 1-very low occupancy & 1.002 & 0.986 to 1.017 & 1.001 & 0.991 to 1.012 \\
\hline & 2-below average occupancy & 1.001 & 0.987 to 1.016 & 1.001 & 0.992 to 1.010 \\
\hline & 3-average occupancy (ref) & 1.000 & & 1.000 & \\
\hline & 4-above average occupancy & 0.990 & 0.976 to 1.004 & 0.993 & 0.984 to 1.003 \\
\hline & 5-very high occupancy & 1.016 & 1.001 to 1.032 & 1.011 & 1.001 to 1.021 \\
\hline \multirow{5}{*}{$\begin{array}{l}\text { Elective day-case inpatient occupancy } \\
\text { quintiles }\end{array}$} & 1-very low occupancy & 0.957 & 0.941 to 0.974 & 0.971 & 0.959 to 0.982 \\
\hline & 2-below average occupancy & 0.964 & 0.949 to 0.980 & 0.976 & 0.965 to 0.987 \\
\hline & 3-average occupancy (ref) & 1.000 & & 1.000 & \\
\hline & 4-above average occupancy & 1.029 & 1.012 to 1.046 & 1.019 & 1.008 to 1.030 \\
\hline & 5-very high occupancy & 1.058 & 1.038 to 1.078 & 1.038 & 1.025 to 1.051 \\
\hline
\end{tabular}

elective and day-case and maternity case are high. However, an admission is less likely when ED occupancy is high, when daycase numbers are low and emergency inpatient occupancy is either very high or very low.

\section{Relation to existing literature}

Our results with respect to admission thresholds when ED is crowded concur with a 2019 US study that found that ED crowding was associated with reduced likelihood of admission. ${ }^{12}$ An earlier US study found no association between crowding and admission thresholds, but this study did not control for a number of important case-mix variables. ${ }^{13}$ Our results with respect to admission thresholds when emergency inpatient occupancy is high concur with the results of a 2014 Swedish study. ${ }^{14}$

\section{Possible mechanisms}

Our observational study offers no direct insight into mechanisms that may underpin these relationships. We can nonetheless hypothesise some mechanisms which appear consistent with the observed data.

The effects of ED crowding on admission thresholds that we have estimated are independent of inpatient occupancy levels. So although ED crowding might often coincide with high inpatient occupancy, the ED crowding effect cannot be explained with reference to inpatient occupancy. Reductions in the probability of admission when ED occupancy is high may, however, be explained with reference to attendance duration. As ED occupancy increases, staff to patient ratios fall, and so the time that it takes to manage the components of patient care (triage, tests and investigations, treatments, decision to admit/discharge) increases. As the average duration of patient stays increases, so too does the potential for the results from complex tests to be returned while the patient is still in the department. Given that most test results are negative, this increases the opportunity for clinicians to conclude a patient's treatment pathway without the need for admission. Furthermore, the longer a patient spends in ED the greater the opportunity for their condition to improve of its own accord, although the spike in admissions at the 4-hour target threshold is a well-known artefact.

The observed parabolic-shaped relationship between the probability of admission and emergency inpatient occupancy might be explained by two competing effects. As emergency inpatient occupancy increases, the opportunity cost of admitting a patient also increases. When inpatient bed occupancy is high, for example, clinicians and managers may trade off the benefits of admitting a current patient with that of an unknown patient who might arrive in the next hour (in the circumstances it may be feasible, although suboptimal to complete a patient's care in ED). As such, the value of an empty bed increases as occupancy levels rise, and admission thresholds rise in response. This would fit with Roemer's law which suggests that 'in an insured population, a hospital bed built is a filled bed' ${ }^{15}$

Staff working in ED often request the support of specialists to confirm a diagnosis, and whether admission is required. These specialists balance their time between theatres, wards, clinics and ED. As inpatient occupancy rises, so the availability of specialists to support decisions in ED falls, and without support, ED clinicians may take more risk-averse decisions and admit at lower thresholds. These two effects (bed availability and specialist availability) act in opposite directions. We propose that when bed occupancy is high, the bed availability factor dominates and when bed occupancy is low, the specialist availability factor takes 


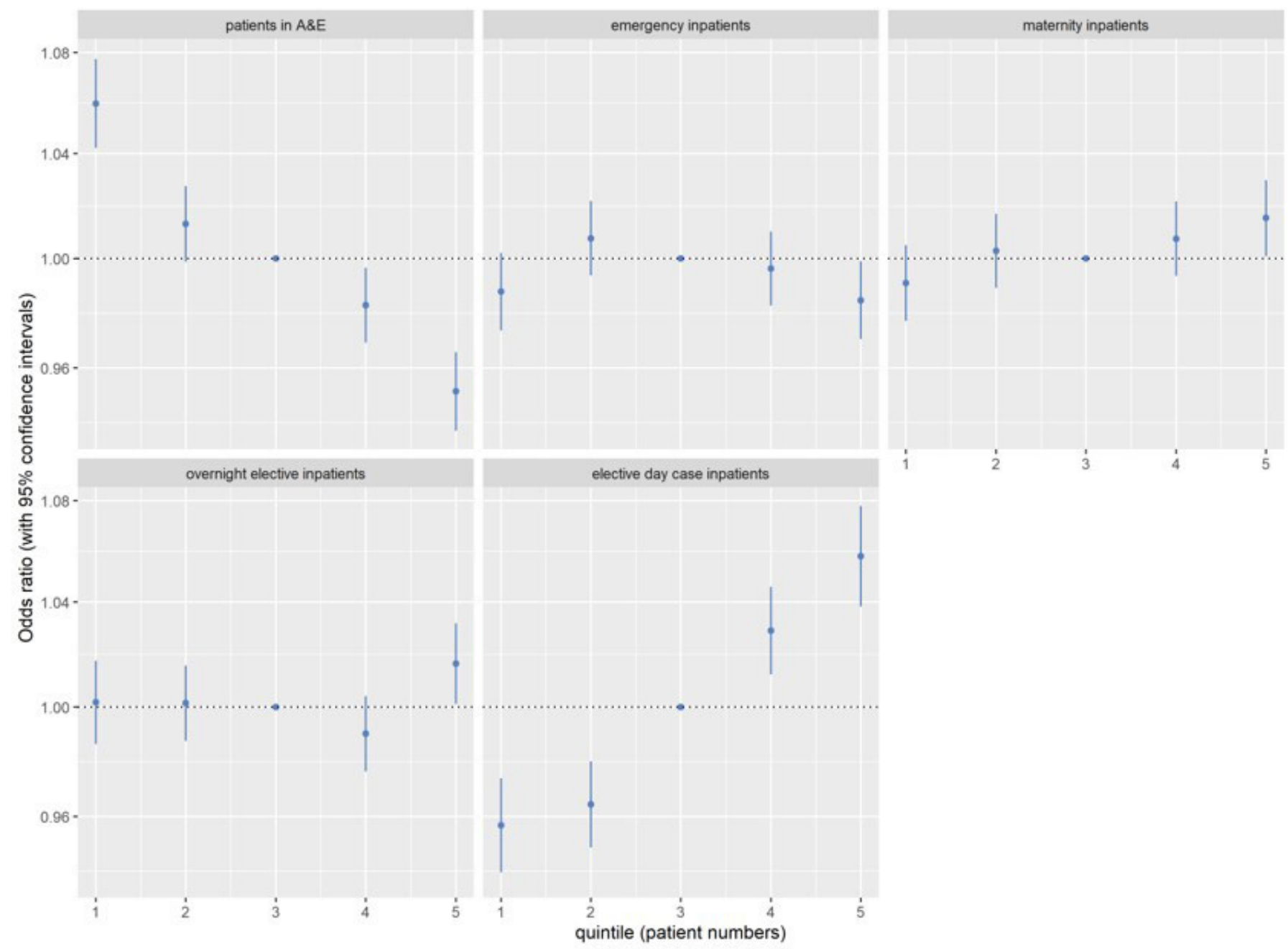

Figure 2 The relationship between ED and inpatient occupancy levels and the probability of admission whiskers denote $95 \%$ Cls.

precedence. In both cases, high and low inpatient occupancy would result in increased admission thresholds.

We found that the probability of admission from ED rose when elective and maternity occupancy rates were high, given the time of day and day of week. While patients in ED tend to not compete for bed space with elective and maternity patients, they may compete for diagnostic capacity. If consultants in ED are unable to rapidly rule out high-risk conditions because diagnostic capacity is being consumed by elective and maternity patients, then they may choose to admit the patient until the test can be carried out.

Qualitative research or more targeted quantitative studies may be able to test these hypotheses and establish the causal mechanisms that underpin our findings.

\section{Limitations}

While our models adjusted for a wide range of patient and attendance characteristics, they did not include any direct measure of patient acuity such as triage score or the national early warning score because such data were not consistently available in our study datasets. Our study is based on attendances at consultant-led EDs in 13 NHS Trusts in 2019. These trusts were selected based on data availability and while attendances at these trusts are broadly similar to attendances in England as a whole in terms of the age and sex profile, there were some notable differences in the ethnicity, deprivation and regional profile. The approach used to determine whether a patient was admitted to hospital following attendance at ED relied on linkage between ED and inpatient datasets. The approach may not deliver accurate results in rare situations when a patient attends ED twice in the same day and is discharged after one of these attendances and admitted after the other.

\section{Implications for policy}

Decisions to admit patients are based on the potential benefits of anticipated clinical interventions, and the potential risks of discharging a patient to a location where medical input may not be readily available. If our proposed mechanisms are valid then admission thresholds may operate at similar levels when emergency inpatient occupancy is low and high, these two circumstances are qualitatively different. Heightened admission thresholds when emergency inpatient occupancy is low may be driven by the increased availability of specialists to advise on admission decisions, whereas heightened admission thresholds when emergency inpatient occupancy is high may be driven by necessity (ie, a lack of available beds). We might therefore expect the quality of admission/discharge decisions to be clinically optimal in times of low emergency inpatient occupancy. The long-term upward trend in acute bed occupancy may therefore give rise to safety concerns.

If the relationship between increased probability of admission and increased elective and maternity occupancy is indeed 
mediated by diagnostic capacity, then efforts to reduce unnecessary admissions from ED would be aided by adequate and dedicated emergency diagnostic capacity.

Many policy initiatives seek to reduce the numbers of patients attending $\mathrm{ED}$, by increasing the proportion of ambulance patients who are treated at scene and by diverting patients to other settings such as walk-in centres and primary care. Our study suggests that if these initiatives are successful then admission thresholds may fall, and the number of admissions may rise, unless other compensating changes are made simultaneously.

Our observational study is unable to determine whether the relationship between occupancy and admission thresholds is causal. If however, the relationship is causal, and providers are able to actively manage ED and inpatient occupancy levels (eg, by adopting strategies to discharge more patients before noon), then this might provide a mechanism to safely reduce emergency admissions. With these caveats in mind, our analysis suggests that $5.5 \%$ of admissions might be avoided if hospitals could replicate occupancy conditions that are most conducive to safe discharge from ED.

Contributors SW conceived and conducted the analysis. JMM assembled and sought permissions to share datasets for a preliminary analysis. RJ, JMM and MAM reviewed and commented on the interim findings. SW produced the draft paper and RJ, JMM and MAM offered comments. MAM revised the final draft of the paper. All authors reviewed and agreed on amendments following peer review.

Funding The authors have not declared a specific grant for this research from any funding agency in the public, commercial or not-for-profit sectors.

Competing interests None declared.

Patient consent for publication Not required.

Provenance and peer review Not commissioned; externally peer reviewed.

Data availability statement Data may be obtained from a third party and are not publicly available. Data used in this study were obtained from the National Commissioning Data Repository (NCDR) administered by NHS England. Access to these datasets for individuals not employed by NHS England is controlled by NHS Digital.

Supplemental material This content has been supplied by the author(s). It has not been vetted by BMJ Publishing Group Limited (BMJ) and may not have been peer-reviewed. Any opinions or recommendations discussed are solely those of the author(s) and are not endorsed by BMJ. BMJ disclaims all liability and responsibility arising from any reliance placed on the content. Where the content includes any translated material, BMJ does not warrant the accuracy and reliability of the translations (including but not limited to local regulations, clinical guidelines, terminology, drug names and drug dosages), and is not responsible for any error and/or omissions arising from translation and adaptation or otherwise.

Open access This is an open access article distributed in accordance with the Creative Commons Attribution Non Commercial (CC BY-NC 4.0) license, which permits others to distribute, remix, adapt, build upon this work non-commercially, and license their derivative works on different terms, provided the original work is properly cited, appropriate credit is given, any changes made indicated, and the use is non-commercial. See: http://creativecommons.org/licenses/by-nc/4.0/.

ORCID iD

Steven Wyatt http://orcid.org/0000-0002-3332-0866

\section{REFERENCES}

1 Health \& Social Care Information Centre. Hospital episode statistics - admitted patient care, England - 2014-15. Available: https://files.digital.nhs.uk/ publicationimport/pub19xxx/pub19124/hosp-epis-stat-admi-summ-rep-2014-15-rep. pdf [Accessed Jan 2015].

2 Wyatt S, Child K, Hood A, et al. Changes in admission thresholds in English emergency departments. Emerg Med J 2017;34:773-9.

3 Boyle AA, Weber EJ. Are rising admission thresholds good medicine? Emerg Med $\mathrm{J}$ 2017:34:771-2.

4 England NHS. Average daily available and occupied beds Timeseries Q1 2010/11 to Q2 2019/20. Available: https://www.england.nhs.uk/statistics/wp-content/uploads/ sites/2/2019/11/Beds-Timeseries-2010-11-onwards-Q2-2019-20-ADJ-for-missingsgh54g-1.xls [Accessed 21 Nov 2019].

5 McKenna P, Heslin SM, Viccellio P, et al. Emergency department and hospital crowding: causes, consequences, and cures. Clin Exp Emerg Med 2019:6:189-95.

6 Public Health England. NHS acute (Hospital) trust catchment populations (2020 rebase experimental statistics), V1.1, 2020. Available: https://app.powerbi.com/view? r=eyJrljoiODZmNGQOYzItZDAwZi00MzFiLWE4NzAtMzVmNTUwMThmMTVIliwidCI6ImVINGUxNDk5LTRhMzUtNGIyZS1hZDQ3LTVmM2NmOWRIODY2NilsImMiOjh9 [Accessed 20 Nov 2020].

7 England NHS. NCDR reference library. Available: https://data.england.nhs.uk/ncdr/ database/ [Accessed 20 Nov 2020].

8 Department for Communities and Local Government,. The English indices of deprivation 2015, technical report. Available: https://assets.publishing.service.gov.uk/ government/uploads/system/uploads/attachment_data/file/464485/English_Indices_ of_Deprivation_2015_-_Technical-Report.pdf [Accessed Sep 2015].

9 Hastie T, Tibshirani R. General additive models. In: Statistical science., 1986: 3, 297-310.

10 Grant RL. Converting an odds ratio to a range of plausible relative risks for better communication of research findings. BMJ 2014;348:f7450.

11 R Core Team. R: a language and environment for statistical computing. Vienna, Austria: R Foundation for Statistical Computing, 2018. https://www.R-project.org/

12 Abir M, Goldstick JE, Malsberger R, et al. Evaluating the impact of emergency department crowding on disposition patterns and outcomes of discharged patients. Int J Emerg Med 2019;12.

13 Albrecht $\mathrm{R}$, Jacoby J, Heller M, et al. Do emergency physicians admit more or fewer patients on busy days? J Emerg Med 2011;41:709-12.

14 Blom MC, Jonsson F, Landin-Olsson M, et al. The probability of patients being admitted from the emergency department is negatively correlated to in-hospital bed occupancy - a registry study. Int J Emerg Med 2014;7:8

15 Roemer MI. Obituary: Milton I. Roemer, pioneering UCLA health services Professor and professional who defined health policy in U.S., abroad. University of California Los Angeles School of Public Health, 2001. 